\title{
Dynamic contrast-enhanced breast MRI at 7T and 3T: an intra-individual comparison study
}

\author{
Gisela L. G. Menezes*, Bertine L. Stehouwer, Dennis W. J. Klomp, Tijl A. van der Velden, \\ Maurice A. A. J. van den Bosch, Floortje M. Knuttel, Vincent O. Boer, \\ Wybe J. M. van der Kemp, Peter R. Luijten and Wouter B. Veldhuis
}

\begin{abstract}
The aim of this study is to compare the current state of lesion identification, the BI-RADS classification and the contrast-enhancement behavior at 7T and 3T breast MRI in the same patient group. Twenty-seven patients with thirty suspicious lesions were selected for this prospective study and underwent both 7T and 3T MRI. All examinations were rated by two radiologists (R1 and R2) independently on image quality, lesion identification and BI-RADS classification. We assessed sensitivity, specificity, NPV and PPV, observer agreement, lesion sizes, and contrast-enhancement-tonoise ratios (CENRs) of mass lesions. Fifteen of seventeen histopathological proven malignant lesions were detected at both field strengths. Image quality of the dynamic series was good at 7T, and excellent at 3T ( $P=0.001$ for R1 and $P=0.88$ for R2). R1 found higher rates of specificity, NPV and PPV at 7T when compared to 3T, while R2 found the same results for sensitivity, specificity, NPV and PPV for both field strengths. The observers showed excellent agreement for BI-RADS categories at $7 \mathrm{~T}(K=0.86)$ and $3 \mathrm{~T}(\mathrm{~K}=0.93)$. CENRs were higher at $7 \mathrm{~T}(P=0.015)$. Lesion sizes were bigger at 7T according to R2 $(P=0.039)$. Our comparison study shows that 7T MRI allows BI-RADS conform analysis. Technical improvements, such as acquisition of T2w sequences and adjustment of B1+ field inhomogeneity, are still necessary to allow clinical use of 7 T breast MRI.
\end{abstract}

Keywords: 7T, 3T, Breast MRI, Breast cancer, BI-RADS, Ultra-high field MRI

\section{Background}

Dynamic contrast-enhanced magnetic resonance imaging (DCE-MRI) of the breast has become a wellestablished imaging method for detection of breast carcinomas, and an increased use of 1.5 tesla $(\mathrm{T})$ and $3 \mathrm{~T}$ DCE-MRI systems has been observed over the past decades. Compared to conventional mammography and ultrasound, DCE-MRI is more accurate in detecting multifocal, multicentric and contralateral disease, in assessing the response to neoadjuvant chemotherapy and in providing preoperative staging (Peters et al. 2008). When compared to 1.5T, a higher field strength (3T) showed to have increased signal-to-noise-ratio (SNR), higher

\footnotetext{
*Correspondence: G.DeLimaGomesdeMenezes@umcutrecht.nl Department of Radiology and Nuclear Medicine, University Medical Centre Utrecht, P.O. Box 85500, 3508 GA Utrecht, The Netherlands
}

spatial and temporal resolution (Rahbar et al. 2013, 2015). The $3 \mathrm{~T}$ field also showed differential effects on T1 relaxation times of non-enhancing compared to gadolinium-enhancing tissue, which results in better contrast resolution of the enhancing lesions (Rahbar et al. 2013, 2015; Soher 2007). Recently, there has been a growing interest in investigating the potential role of 7T MRI in breast cancer diagnosis and management (Menezes et al. 2014; Pinker et al. 2014; Stehouwer et al. 2013b; Umutlu et al. 2011). Moving to 7T not only increases SNR (Brown et al. 2013; Korteweg et al. 2011) (and also spatial resolution), (van de Bank et al. 2013) but also brings a greater spectral dispersion, significantly improving magnetic resonance spectroscopy (MRS) (Klomp et al. 2011). Considering these new possibilities, the focus of breast imaging research at $7 \mathrm{~T}$ is not only improving morphology

\section{黛 Springer}

(c) 2016 Menezes et al. This article is distributed under the terms of the Creative Commons Attribution 4.0 International License (http://creativecommons.org/licenses/by/4.0/), which permits unrestricted use, distribution, and reproduction in any medium, provided you give appropriate credit to the original author(s) and the source, provide a link to the Creative Commons license, and indicate if changes were made. 
assessment of breast lesions, but also moving towards obtaining metabolic and cellular information (Klomp et al. 2011; Loo et al. 2011; van der Kemp 2014)

There are also drawbacks of 7T, such as a greater heterogeneity of the static magnetic field (B0) and of the applied RF field $(\mathrm{B} 1+)$. Furthermore, breast coils had to be developed since none were commercially available a decade ago when 7T became available for wholebody imaging. In literature the first attempt to perform DCE-MRI breast imaging at 7T was made by Umutu et al. using a single loop coil, showing the complexity of ultra-high field breast MRI. Only moderate image quality was achieved when using this coil (Umutlu et al. 2011). Subsequent improvements in hardware and imaging strategies have led to an improved image quality and showed that 7T breast MRI is amenable to BI-RADS lexicon conform analysis (Stehouwer et al. 2013a, b). Nowadays, the first bilateral set-ups are available, which illustrates the evolution of 7T towards clinical usage (Brown et al. 2014; Gruber et al. 2014; Pinker et al. 2014; Stehouwer et al. 2013a; van der Velden 2014). Apart from the new contrast mechanisms available at 7T, such as chemical exchange saturation transfer (CEST) and ${ }^{31}$ phosphorous magnetic resonance spectroscopy ( $\left.{ }^{31} \mathrm{P}-\mathrm{MRS}\right)$, the conventional breast MRI still needs to at least offer comparable imaging results to the current clinical standard of 3T imaging to maintain good medical care.

Comparisons of 7T versus breast imaging at lower field strengths are scarce. So far, studies with healthy volunteers imaged using T1-weighted (T1w) non contrastenhanced imaging presented similar or better results for $7 \mathrm{~T}$. The $7 \mathrm{~T}$ images showed increased SNR, better fat-water contrast measures and better objective image quality scores (Brown et al. 2013; Umutlu et al. 2011; van der Velden 2014). In a patient setting, using DCE-MRI, comparisons between $7 \mathrm{~T}$ and $3 \mathrm{~T}$ fields have been made only in one patient case (Stehouwer et al. 2013a), and in one patient study (Brown et al. 2013). These papers suggest that DCE-MRI at 7T is technically feasible (Stehouwer et al. 2013a), and it can provide a high sensitivity and specificity using high temporal and spatial resolution imaging (Brown et al. 2013).

The aim of this study is to compare the current state of lesion identification and BI-RADS classification at DCEMRI at $7 \mathrm{~T}$ and $3 \mathrm{~T}$ in the same patient group.

\section{Results}

\section{Study population}

Thirty breast lesions were reported in 27 patients. All lesions were detected on conventional imaging, and were classified as BI-RADS $4(\mathrm{n}=21)$ and BI-RADS $5(\mathrm{n}=9)$. The mean age was 55 years (SD 8; range $32-74$ years). Of the 30 index lesions, 17 were histopathologically malignant, and 13 were benign (Table 1 ).

In 8 patients the 7T scan was conducted using a unilateral breast coil and in 19 using a bilateral breast coil. Fifteen women were postmenopausal. The remaining 11 patients were imaged with a mean of 3.4 days between examinations.

\section{Image assessment}

Both observers detected, at both field strengths, 15 of 17 malignant index. Both observers classified 11 lesions as mass, 2 as non-mass-like-enhancement and 2 as architectural distortions without enhancement. One pure DCIS and one IDC were not detected at either field strength. The detected malignant index lesions and BI-RADS

Table 1 Characteristics of the 27 patients with 30 suspicious breast lesions detected on conventional breast imaging

\begin{tabular}{|c|c|}
\hline \multicolumn{2}{|l|}{ Characteristics } \\
\hline Age (years) & Mean: 55 (SD: 8, range: 32-74) \\
\hline \multicolumn{2}{|l|}{ Presentation on conventional imaging } \\
\hline Mass & $15(50 \%)$ \\
\hline Calcifications & $11(37 \%)$ \\
\hline Mass + calcifications & $1(3 \%)$ \\
\hline Architectural distortion & $1(3 \%)$ \\
\hline Architectural distortion + calcifications & $2(7 \%)$ \\
\hline $\begin{array}{l}\text { Lesion size on conventional imaging } \\
(\mathrm{mm})\end{array}$ & mean: 19 (SD: 9, range: 7-39) \\
\hline \multicolumn{2}{|l|}{$\begin{array}{l}\text { BI-RADS category on conventional } \\
\text { imaging per patient }\end{array}$} \\
\hline 4 & $21(70 \%)$ \\
\hline 5 & $9(30 \%)$ \\
\hline \multicolumn{2}{|l|}{ Histological type } \\
\hline Malignant & $17(57 \%)$ \\
\hline Invasive ductal carcinoma & 5 \\
\hline Invasive lobular carcinoma & 1 \\
\hline Invasive ductulolobular carcinoma & 7 \\
\hline $\begin{array}{l}\text { Ductal carcinoma in situ with invasive } \\
\text { component }\end{array}$ & 2 \\
\hline Ductal carcinoma in situ & 2 \\
\hline Non-malignant & $13(43 \%)$ \\
\hline Columnar cell lesion & 2 \\
\hline Radial scar lesion & 1 \\
\hline Atypical ductal hyperplasia & 1 \\
\hline Cyst & 1 \\
\hline Hamartoma & 1 \\
\hline Fibroadenoma & 2 \\
\hline Apocrine metaplasia & 2 \\
\hline Fibrocystic changes & 2 \\
\hline Sclerosing adenosis & 1 \\
\hline
\end{tabular}

a Lesion size of the 15 mass lesions 
descriptors are presented in Table 2. The table also illustrates the similarities and differences in ratings between field-strengths for both observers. Figure 1 shows one example of a patient with a malignant lesion.

All patients and identified lesions are presented in Table 3 (with their respective BI-RADS classification and histopathological results).

In 3 of the 13 non-malignant lesions, a BI-RADS 4 or higher was rated at one or both field strengths. In case number 8 (Table 3), a radial scar lesion was rated as BIRADS 4 or 5 by both observers at both field strengths. In case number 12, R1 identified a non-mass-like enhancement at 3T (rated as BI-RADS 4). The same lesion was not identified by this observer at 7T. R2 identified a nonmass-like enhancement at 7T (BI-RADS 4), and a mass lesion at 3T (BI-RADS 5). In case number 19, R1 identified a non-mass-like enhancement lesion at 7T (BIRADS 4), and a mass and non-mass-like enhancement at 3T (also rated as BI-RADS 4). R2 identified non-masslike enhancement at both field strengths and classified both as BI-RADS 3 . The remaining 10 cases with proven benign index lesions had examinations that were correctly classified as benign at both field strengths. Figure 2 shows an example of a benign lesion.

Table 3 also shows that a number of additional findings were made independently of the index lesions. These findings (in italic) occurred in five cases, and were all classified as benign. Figure 3 shows an example of benign additional finding (periductal enhancement), classified as BI-RADS 1-3 by both observers. The final histopathological analysis showed a cyst.

According to the first observer (R1), the mean image quality score was $2.14 \pm 0.82$ for $7 \mathrm{~T}$ images, and $1.37 \pm 0.49$ for $3 \mathrm{~T}$. The second observer (R2) scored image quality as $1.96 \pm 0.65$ for $7 \mathrm{~T}$, and $1.70 \pm 0.60$ for $3 \mathrm{~T}(\mathrm{P}=0.001$ for $\mathrm{R} 1$, and $\mathrm{P}=0.88$ for $\mathrm{R} 2)$. Image quality scores were also calculated considering only the scans performed with the bilateral coil. We had similar results: R1 rated the image quality of the dynamic series significantly better at 3T $(P=0.021)$, and $R 2$ saw no significant difference between fields $(\mathrm{P}=0.132)$. Image quality scores are presented in Table 4.

Table 5 shows sensitivity, specificity, NPV and PPV at $3 \mathrm{~T}$ and $7 \mathrm{~T}$ for both observers. The rates were calculated for the 19 patients with bilateral coil and for the total number of patients (27).

Inter-observer agreement for BI-RADS assessment categories was excellent in both $7 \mathrm{~T}(\mathrm{~K}=0.93$ and $\mathrm{P}=0.0001)$ and $3 \mathrm{~T}(\mathrm{~K}=0.86$ and $\mathrm{P}=0.0001)$.

The 11 malignant mass lesions had a mean size of $31 \mathrm{~mm}$ at 7T MRI according to R1 (SD 18, range 16-95) and a mean size of $28 \mathrm{~mm}$ at 3T MRI (SD 23, range 10-95). For $\mathrm{R} 2$ the mean size at $7 \mathrm{~T}$ was $28 \mathrm{~mm}$ (SD 16, range 15-72), and at $3 \mathrm{~T}$ was $24 \mathrm{~mm}$ (SD 13, range 12-58). The differences between field strengths were not statistically significant for $\mathrm{R} 1(\mathrm{P}=0.864)$, but according to $\mathrm{R} 2$ lesions sizes were significantly bigger at $7 \mathrm{~T}(\mathrm{P}=0.039)$. Figure 4 shows a patient case in which the size of the tumor was much more clearly depicted at $7 \mathrm{~T}$ when compared to $3 \mathrm{~T}$.

CENRs measurements of the malignant mass lesions showed to be significantly higher at $7 \mathrm{~T}(\mathrm{P}=0.015)$ with a mean of 2.8 (SD 1.0, range 1.4-4.6), compared to 2.0 at 3T (SD 0.6, range 1.2-3.4).

\section{Discussion}

The performance in this study of both 7T and 3T DCEMRI (using conventional imaging parameters) is conform literature (Peters et al. 2008). Sensitivity, specificity, NPV and PPV were equal at both field strengths for R2. However, R1 achieved higher specificity, NPV and PPV rates at $7 \mathrm{~T}$ (considering both coils and only bilateral coil). These results could be related to the simultaneous high spatial and temporal resolution provided at $7 \mathrm{~T}$ when compared to the resolution obtained at 3T. Our findings may be somewhat limited by the small sample size, though similar results have previously been described. Gruber et al. had similar outcomes to ours. The authors compared bilateral DCE-MRI of the breast at 3T and 7T in 24 patients and the results showed higher sensitivity, PPV and NPV at 7T. They attributed these results to high temporal and spatial resolution provided at the ultrahigh magnetic field (Gruber et al. 2014).

In prospective studies, Pinker et al. and Kuhl et al. analyzed the trade-off between temporal and spatial resolution in dynamic post-contrast bilateral MR imaging of the breast. Both authors concluded that, at lower field strengths, the SNR is not sufficient to acquire images at higher spatial resolution, even when applying acceleration techniques to characterize the breast lesions (Kuhl et al. 2005; Pinker et al. 2009). Recently, van den Bank et al. hypothesized that the necessary gain in SNR could be obtained at 7T field, while acceleration could be obtained with high-density receiver coil arrays. Using a unilateral 30-channel receive-only element breast coil (combined with a dual-channel transmit coil) the authors concluded that the high density of receive coil elements allowed high temporal and spatial resolution DCE-MRI of breast at 7T, associated with less DWI distortion (van de Bank et al. 2013).

The ultra-high resolution imaging at $7 \mathrm{~T}$ performed in our study allowed better morphological characterizations of malignant breast tumors when compared to 3T, as illustrated in Figs. 1 and 4. In Fig. 4 the lesions' dimensions are more clearly depicted in ultra-high resolution 7T images when compared to 3T. At 3T, the spiculated lesion would be classified as a T1 (tumor is less than 


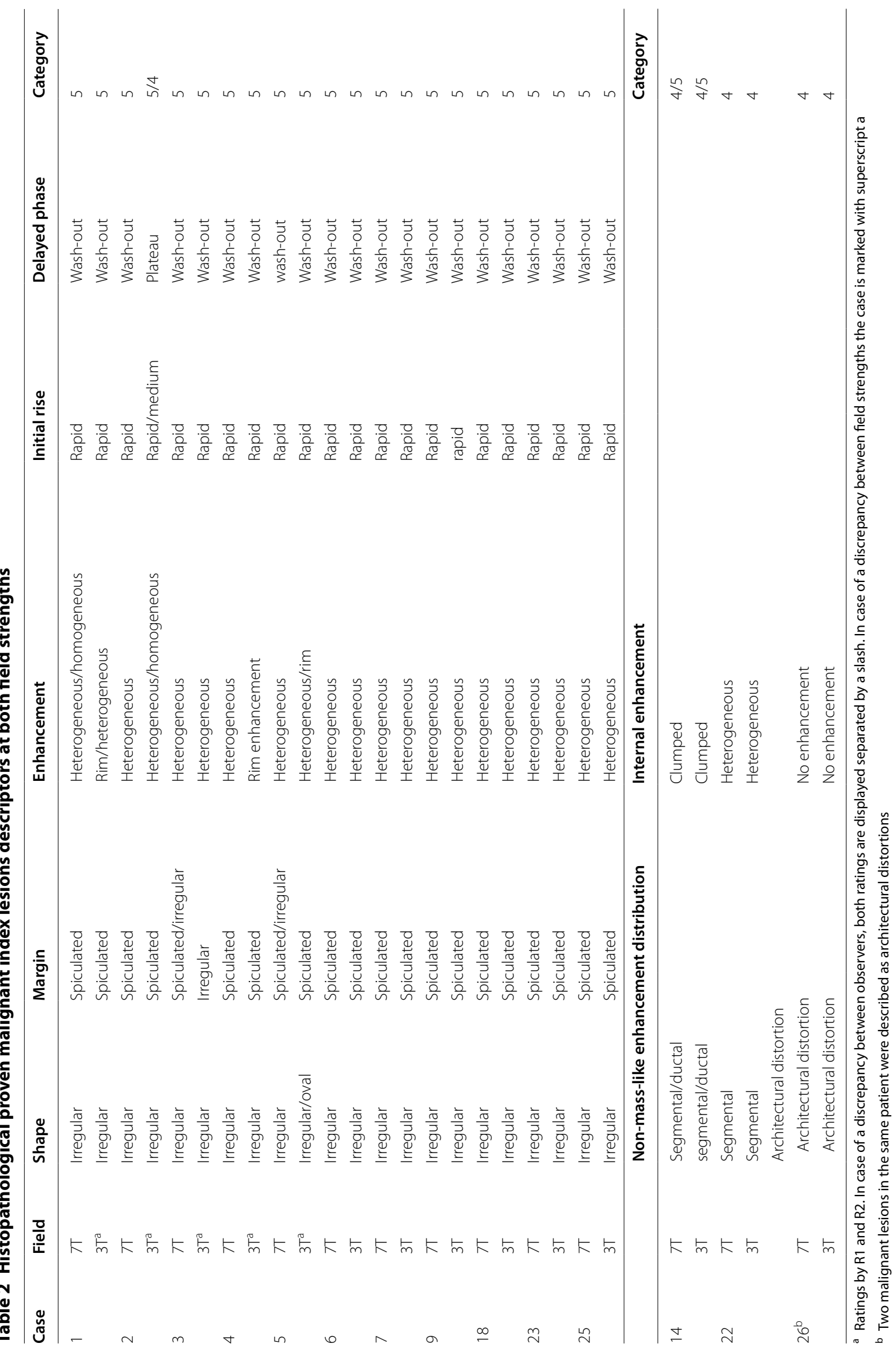



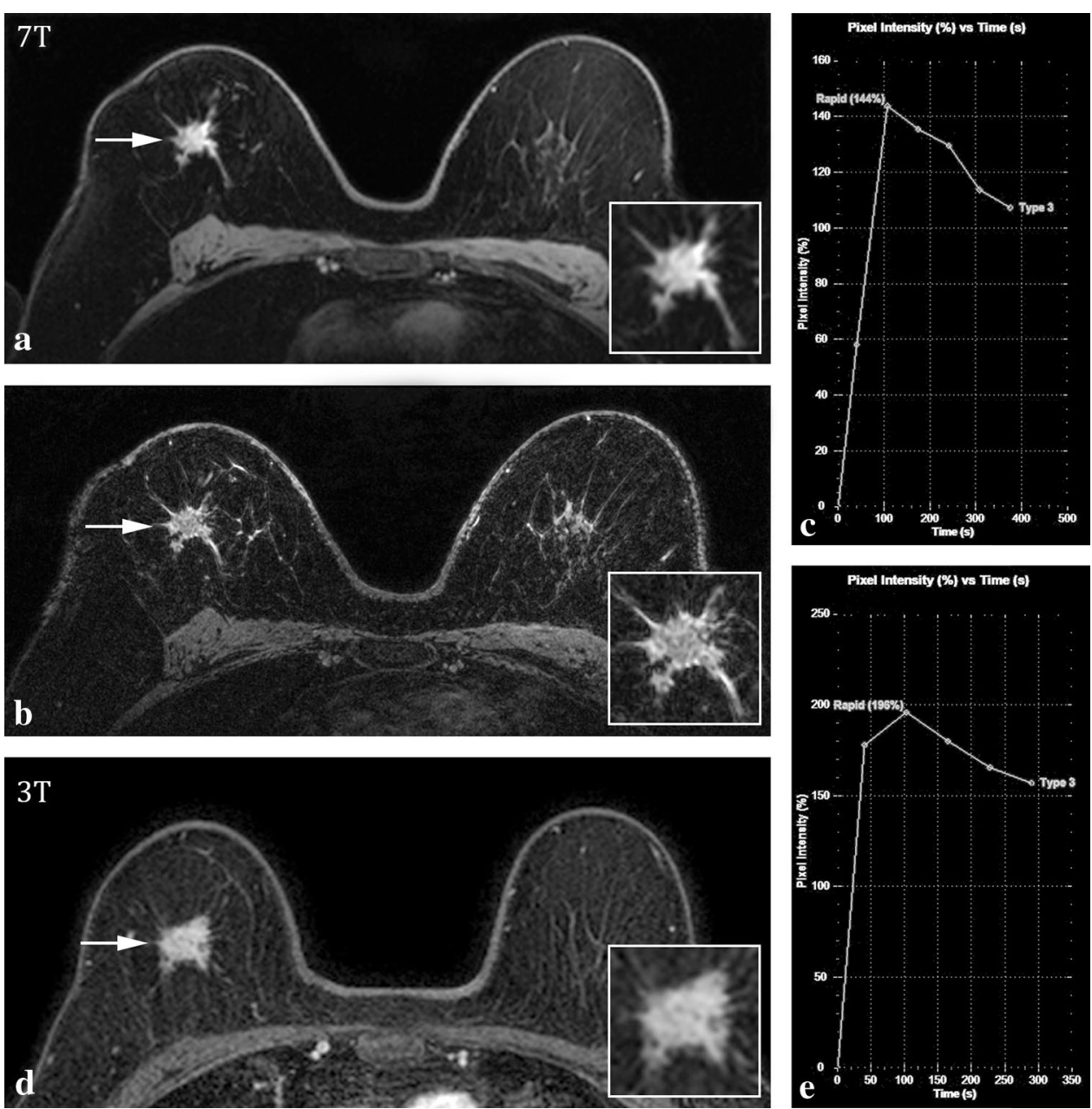

Fig. $17 T(\mathbf{a}-\mathbf{c})$ and 3T (d, e) MRI images of a 67-year-old female with an invasive lobular carcinoma in her right breast. Transverse image of 2nd post contrast-injection series (a, d) shows an irregular mass lesion with spiculated margins (arrows) on both field strengths. Inset shows zoomed-in image. Ultra-high resolution 7T image of the same slice $(\mathbf{b})$. The kinetic curve assessment showed an initial rapid rise and wash-out pattern in the delayed phase on both field strengths (c, e). Both observers rated the lesion as BI-RADS 5

$2 \mathrm{~cm}$ ) and at 7T it would be classified as T2 (between 2 and $5 \mathrm{~cm}$ ). This difference upgrades the cancer from stage I to stage II, which results in an impact in treatment and, ultimately, in prognosis.

The image quality for the dynamic series at $7 \mathrm{~T}$ was overall rated good by both observers. Even though R1 achieved higher specificity, PPV and NPV rates at $7 \mathrm{~T}$, the same observer rated the image quality of the dynamic series at $3 \mathrm{~T}$ significantly better, which indicates that there is still room for improvement. Current technical developments, such as dynamic B0 field monitoring and correction (Boer et al. 2012), and RF pulses designed to compensate the linear decreasing B1+ field(van Kalleveen et al. 2015) may improve the image quality at 7T. As an example, Boer et al. developed a simple field probe that proved to be useful to monitor temporal B0 field variations. The acquired temporal B0 field information could drive a dynamic module to correct the B0 magnetic field in real time (Boer et al. 2012). In a recent study, van Kalleveen et al. were able to compensate the inhomogeneous B1+ field (while keeping the specific absorption rate low) by applying tilt optimized flip uniformity RF pulses to the breast surface coil (van Kalleveen et al. 2015).

Brown et al. performed two studies in healthy volunteers, and the results showed equal image quality for non-contrast-enhanced T1w imaging at 7T and 3T MRI (Brown et al. 2013, 2014). In these studies, SPAIR was used as fat suppression technique, which is less prone to B0 field inhomogeneities than a binomial RF pulse. 
Table 3 Identified mass and non-mass-like enhancement lesions at 7T and 3T plus BI-RADS classification and pathology results

\begin{tabular}{|c|c|c|c|c|c|c|c|c|c|}
\hline Case & R1-7T & BI-RADS & $\mathrm{R} 2-7 \mathrm{~T}$ & BI-RADS & R1-3T & BI-RADS & $\mathrm{R} 2-3 \mathrm{~T}$ & BI-RADS & Pathology \\
\hline 1 & Mass & 5 & Mass & 5 & Mass & 5 & Mass & 5 & IDLC \\
\hline 2 & Mass & 5 & Mass & 5 & Mass & 5 & Mass & 4 & IDLC \\
\hline 3 & Mass & 5 & Mass + non-mass & 5 & Mass & 5 & Mass + non-mass & 5 & IDLC \\
\hline 4 & Mass & 5 & Mass & 5 & Mass & 5 & Mass & 5 & IDLC \\
\hline 5 & Mass + satellite & 5 & Mass & 5 & Mass & 5 & Mass & 5 & IDC \\
\hline 6 & Mass & 5 & Mass & 5 & Mass & 5 & Mass & 5 & IDC \\
\hline 7 & Mass & 5 & Mass & 5 & Mass & 5 & Mass & 5 & IDLC \\
\hline 8 & Mass & 4 & Mass & 4 & Mass & 4 & Mass & 5 & Radial scar lesion \\
\hline 9 & Mass + satellite & 5 & Mass + non-mass & 5 & Mass + non-mass & 5 & Mass & 5 & IDLC \\
\hline 10 & No lesion & 1 & No lesion & 2 & No lesion & 1 & No lesion & 2 & Apocrine metaplasia \\
\hline 11 & No lesion & 3 & Non-mass & 2 & Non-mass & 3 & Non-mass & 3 & Cyst \\
\hline 12 & Mass & 3 & Non-mass & 4 & Non-mass & 4 & Mass & 5 & Fibrocystic changes \\
\hline 13 & No lesion & 2 & No lesion & 2 & No lesion & 1 & No lesion & 2 & DCIS \\
\hline 14 & Non-mass & 4 & Non-mass & 5 & Non-mass & 4 & Non-mass & 5 & $\begin{array}{l}\text { DCIS + invasive } \\
\text { component }\end{array}$ \\
\hline 15 & No lesion & 1 & No lesion & 2 & Non-mass & 3 & No lesion & 2 & Hamartoma \\
\hline 16 & Mass & 2 & Mass $2 \times$ & 3 & No lesion & 2 & Mass $2 \times$ & 3 & Apocrine metaplasia \\
\hline 17 & $\begin{array}{l}\text { Mass } 2 \times+ \text { non- } \\
\text { mass }\end{array}$ & 3 & Mass $2 \times$ & 2 & $2 \times$ Mass & 3 & Mass $2 \times$ & 3 & $\begin{array}{l}\text { Fibrocystic } \\
\text { changes }+ \\
\text { fibroadenoma }\end{array}$ \\
\hline 18 & Mass + non-mass & 5 & Mass & 5 & $2 \times$ Mass & 5 & Mass & 5 & ILC \\
\hline 19 & Non-mass & 4 & Non-mass & 3 & Mass + non-mass & 4 & Non-mass $2 x$ & 3 & Columnar cell lesion \\
\hline 20 & No lesion & 1 & No lesion & 2 & No lesion & 2 & No lesion & 2 & $\begin{array}{l}\text { Columnar cell } \\
\text { lesion + ADH }\end{array}$ \\
\hline 21 & No lesion & 2 & no lesion & 2 & No lesion & 2 & No lesion & 2 & Sclerosing adenosis \\
\hline 22 & Non-mass & 4 & Non-mass & 4 & Non-mass & 4 & Non-mass & 4 & $\begin{array}{c}\text { DCIS + invasive } \\
\text { component }\end{array}$ \\
\hline 23 & Mass & 5 & Mass & 5 & Mass & 5 & Mass & 5 & DCIS \\
\hline 24 & No lesion & 2 & No lesion & 2 & No lesion & 2 & No lesion & 2 & IDC \\
\hline 25 & Mass & 5 & Mass & 5 & Mass & 5 & Mass & 5 & IDC \\
\hline 26 & $\begin{array}{l}\text { Architectural } \\
\text { distortion } 2 \times\end{array}$ & 4 & $\begin{array}{l}\text { Architectural } \\
\text { distortion } 2 \times\end{array}$ & 4 & $\begin{array}{l}\text { Architectural } \\
\text { distortion } 2 \times\end{array}$ & 4 & $\begin{array}{l}\text { Architectural } \\
\text { distortion } 2 \times\end{array}$ & 4 & $\mathrm{IDC}+\mathrm{IDLC}$ \\
\hline 27 & No lesion & 2 & No lesion & 2 & No lesion & 2 & No lesion & 2 & Fibroadenoma \\
\hline
\end{tabular}

Additional findings are marked in italic

However, SPAIR is incompatible with optimal dynamic imaging at $7 \mathrm{~T}$ due to specific absorption rate (SAR) limitations. Other fat suppression techniques (such as a DIXON-based method) could be investigated for $7 \mathrm{~T}$ dynamic imaging (Ma 2008) since it showed promising results at 3T MRI and is less sensitive to B0 distortions (Le-Petross et al. 2010).

In vitro research showed that gadolinium based contrast agents are slightly less effective at $7 \mathrm{~T}$ compared to 3T (Noebauer-Huhmann et al. 2010). However, our results showed a higher CENRs at 7T, and Gruber et al. also reported a non-significant difference in relative signal-enhancement between 3T and 7T (Gruber et al. 2014). It can thus be suggested that, with adequate T1 weighting and the high SNR available at 7T, the effectiveness of the contrast agent is (more than) sufficient. With the higher CENRs, all invasive mass lesions were conspicuous at $7 \mathrm{~T}$.

To our knowledge this is the second cohort study to compare dynamic contrast-enhanced breast MRI at $7 \mathrm{~T}$ and 3T. This is a relatively new subject, therefore we decided to present all information obtained in unilateral and bilateral coils. The use of a unilateral breast coil at $7 \mathrm{~T}$ in the first 8 patients was subsequently replaced by a bilateral breast coil. This transition was a step forward towards clinical usage. However, further improvements are still necessary. By including multiple receiver elements in the bilateral coil, (van der Velden 2014) imaging with a high spatial and temporal resolution can be combined with the use of parallel imaging techniques. As 

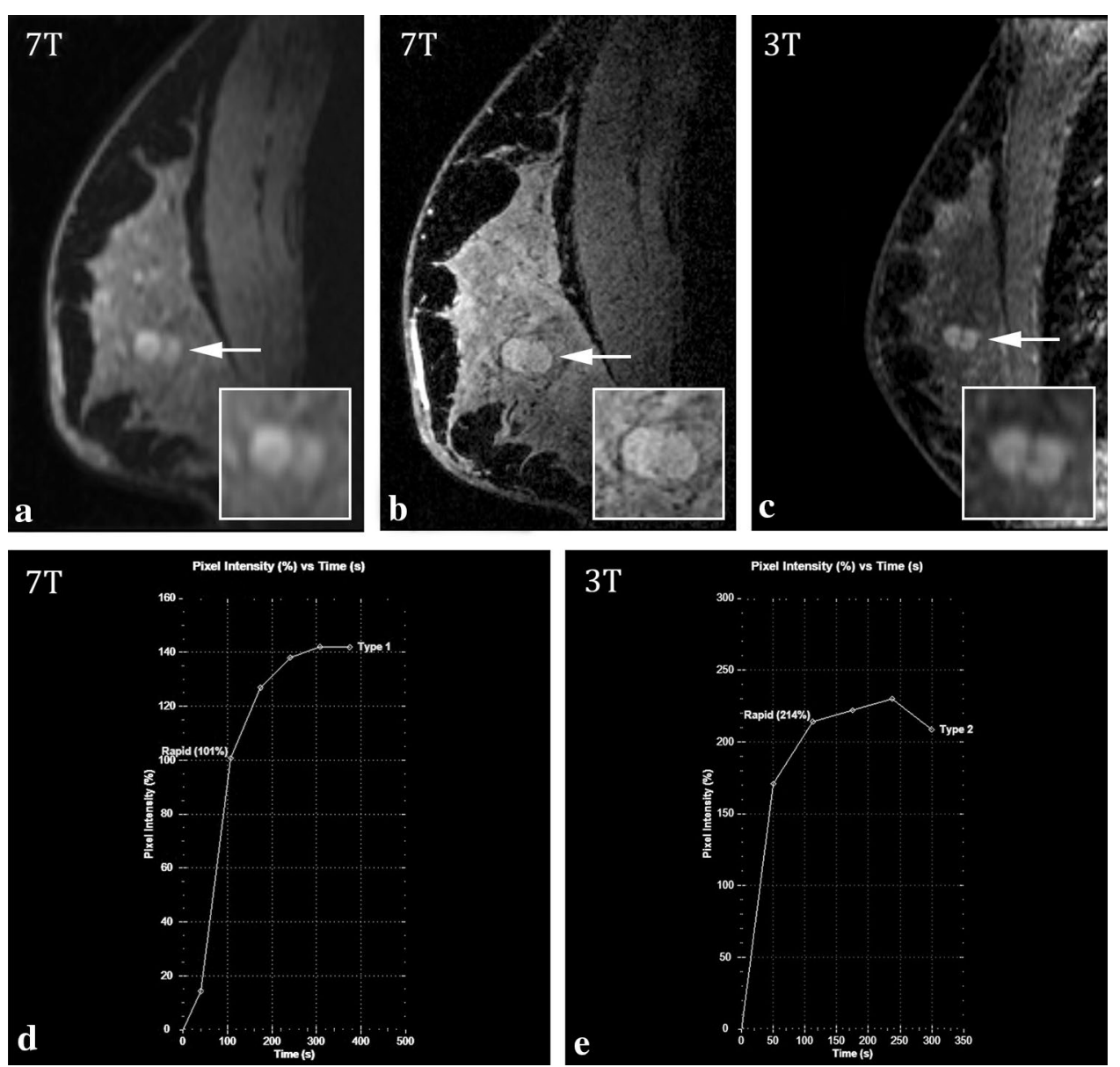

Fig. $27 T(\mathbf{a}, \mathbf{b}, \mathbf{d})$ and $3 T(\mathbf{c}, \mathbf{e})$ MRI images of a 65-year-old female patient. The depicted lesion in her right breast was diagnosed as fibrocystic changes after biopsy. Sagittal images of 2nd post contrast-injection series (a, c) show a lobular lesion (arrow) with irregular (R1 at 3T) or smooth margins (R1 at 7T and R2 at 3T and 7T). Inset shows zoomed-in image. Ultra-high resolution 7T image of the same slice (b). The kinetic curve assessment at $7 \mathrm{~T}$ shows a rapid rise and persistent pattern in the delayed phase (d), and at $3 \mathrm{~T}$ a rapid rise and plateau pattern (e). R1 classified the lesion as BI-RADS 3, and R2 as BI-RADS 3 (3T) and BI-RADS 2 (7T)

mentioned previously, the feasibility of the implementation of a multiple receiver array has been shown by van den Bank et al. using a unilateral breast coil (van de Bank et al. 2013) and recently by Brown et al. in a bilateral setup (Brown et al. 2013, 2014).

One limitation of our study is that $\mathrm{T} 2 \mathrm{w}$ images were not acquired at 7T (and neither was in previous 7T breast MRI studies) (Brown et al. 2013; Gruber et al. 2014; Stehouwer et al. 2013a, b, 2014). Due to the use of multiple refocusing pulses, T2w sequences based in turbo spin echo have significant $\mathrm{B} 1+$ problems. Moreover, the association of the same multiple refocusing pulses and inversion recovery increases the SAR substantially (which is already close to the SAR limit at 3T). Fat-suppressed T2w sequences also have severe limitations (Gruber et al. 2014; Stehouwer et al. 2013a, b). Although the latest update of the
BI-RADS lexicon recommends to include T2w imaging in the breast imaging protocol, (Edwards 2013) the most recent published $\mathrm{T} 2 \mathrm{w}$ data at $7 \mathrm{~T}$ do not meet imaging standards, (Umutlu et al. 2011) once we lack the necessary homogeneous distribution of $180^{\circ}$ flip angles. Current developments such as RF pulses designed to compensate the linear decreasing B1+ field may enable accurate T2w imaging at 7T (van Kalleveen et al. 2015). However, further research and technical improvements are necessary (Gruber et al. 2014; van Kalleveen et al. 2015).

Another limitation is the small sample size. The first cohort study comparing breast MRI at 7T and 3T had a similar number of patients (24). Nevertheless, the presented lesions in our study encompassed a wide range of pathological diagnoses, including both benign and high risk lesions, and both in situ and invasive cancers. 

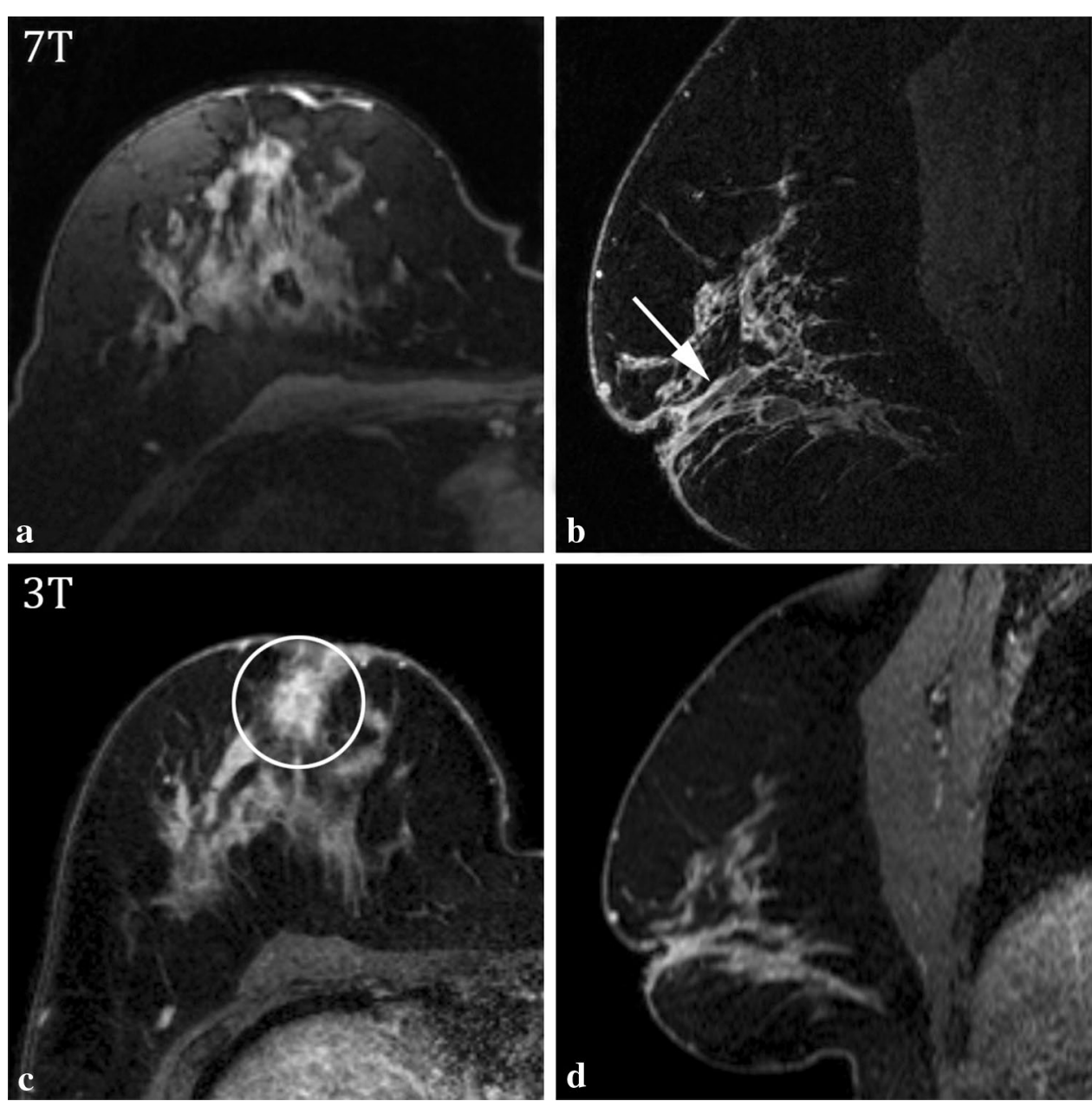

Fig. $37 T(\mathbf{a}, \mathbf{b})$ and $3 T(\mathbf{c}, \mathbf{d})$ MRI results of a 47-year-old female patient with a history of inverted nipples. The biopsied index lesion in the right breast showed to be a cyst. Transverse image of 2 nd post contrast-injection series (a, c). 7T MRI sagittal slice of high-resolution imaging (b), sagittal slice of 3T dynamic series at approximately the same location (d). At 7T MRI, diffuse non-mass-like enhancement was identified by R2, while R1 identified periductal enhancement (arrow). At 3T MRI, a focal non-mass-like enhancement was identified by R1 (circle), and multiple regions of nonmass-like enhancement were seen by R2. The observers rate the images BI-RADS 3 for 3 T MRI, and BI-RADS 3 (R1) and 2 (R2) for 7T MRI

Table 4 Image quality assessment of the 27 dynamic series rated by $R 1$ and $R 2$ at both field strengths

\begin{tabular}{lllll}
\hline & R1* & & R2 & \\
\hline Image quality & $7 \mathrm{~T}$ & $3 \mathrm{~T}$ & $7 \mathrm{~T}$ & $3 \mathrm{~T}$ \\
Excellent & 7 & 17 & 6 & 10 \\
Good & 9 & 10 & 16 & 15 \\
Moderate & 11 & 0 & 5 & 2 \\
Poor & 0 & 0 & 0 & 0 \\
Non-diagnostic & 0 & 0 & 0 & 0 \\
\hline
\end{tabular}

* $<0.05$ Wilcoxon matched pairs signed rank test

\section{Conclusion}

Our comparison study shows that 7T DCE breast MR allows BI-RADS conform analysis. However, technical improvements are needed (such as acquisition of T2w sequences and adjustment of B1+ field inhomogeneities) to further explore the clinical potential of 7T breast MRI.

\section{Methods}

\section{Study population}

The study population consisted of two groups. The first group consisted of female patients selected from 
Table 5 Sensitivity, specificity, NPV and PPV at 3T and 7T for both observers (R1 and R2)

\begin{tabular}{|c|c|c|c|c|}
\hline & \multirow[t]{2}{*}{ Sensitivity } & \multicolumn{2}{|c|}{ R1 unilateral + bilateral coil $(n=27)^{a}$} & \multirow[t]{2}{*}{ NPV } \\
\hline & & Specificity & PPV & \\
\hline $3 \mathrm{~T}$ & $88 \%$ (Cl 0.62-0.98) & $77 \%(\mathrm{Cl} 0.46-0.94)$ & $83 \%(\mathrm{Cl} 0.58-0.96)$ & $83 \%(\mathrm{Cl} 0.51-0.97)$ \\
\hline \multirow[t]{3}{*}{$7 \mathrm{~T}$} & $88 \%(\mathrm{Cl} 0.62-0.98)$ & $85 \%(\mathrm{Cl} 0.54-0.97)$ & $88 \%(\mathrm{Cl} 0.62-0.98)$ & $85 \%(C l 0.54-0.97)$ \\
\hline & Sensitivity & \multicolumn{2}{|c|}{ R1 bilateral coil $(n=19)^{b}$} & \multirow[t]{2}{*}{ NPV } \\
\hline & & Specificity & PPV & \\
\hline $3 T$ & $80 \%$ (Cl 0.44-0.96) & $83 \%(\mathrm{Cl} 0.51-0.97)$ & $80 \%(\mathrm{Cl} 0.44-0.96)$ & $83 \%(C l 0.51-0.97)$ \\
\hline \multirow[t]{3}{*}{$7 \mathrm{~T}$} & $80 \%(\mathrm{Cl} 0.44-0.96)$ & $92 \%(C l 0.60-0.99)$ & $89 \%(\mathrm{Cl} 0.51-0.99)$ & $85 \%$ (Cl 0.54-0.97) \\
\hline & Sensitivity & \multicolumn{2}{|c|}{ R2 unilateral + bilateral coil $(n=27)^{a}$} & \multirow[t]{2}{*}{ NPV } \\
\hline & & Specificity & PPV & \\
\hline $3 \mathrm{~T}$ & $88 \%$ (Cl 0.62-0.98) & $85 \%(\mathrm{Cl} 0.54-0.97)$ & $88 \%(\mathrm{Cl} 0.62-0.98)$ & $85 \%(C l 0.54-0.97)$ \\
\hline \multirow[t]{3}{*}{$7 \mathrm{~T}$} & $88 \%(\mathrm{Cl} 0.62-0.98)$ & $85 \%(\mathrm{Cl} 0.54-0.97)$ & $88 \%(\mathrm{Cl} 0.62-0.98)$ & $85 \%(C l 0.54-0.97)$ \\
\hline & Sensitivity & \multicolumn{2}{|c|}{ R2 bilateral coil $(n=19)^{b}$} & \multirow[t]{2}{*}{ NPV } \\
\hline & & Specificity & PPV & \\
\hline $3 T$ & $80 \%(\mathrm{Cl} 0.44-0.96)$ & $92 \%(\mathrm{Cl} 0.6-0.99)$ & $89 \%(C l 0.51-0.99)$ & $85 \%$ (Cl 0.54-0.97) \\
\hline $7 \mathrm{~T}$ & $80 \%(\mathrm{Cl} 0.44-0.96)$ & $92 \%(\mathrm{Cl} 0.6-0.99)$ & $89 \%(C l 0.51-0.99)$ & $85 \%$ (Cl 0.54-0.97) \\
\hline
\end{tabular}

a Scans performed using both coils

b Scans performed using only the bilateral coil

a previously initialized 7T breast MRI feasibility study (Stehouwer et al. 2013b). These patients also underwent 3T MRI scan for clinical indications. The second group consisted of female patients who underwent both $3 \mathrm{~T}$ and 7T MRI for the purpose of intra-individual comparison. In both groups the inclusion criteria were the same: women whose age was $\geq 18$ years and with a suspicious breast lesion (BI-RADS 4 or 5) detected on conventional imaging. Exclusion criteria for both studies were: age $<18$ years, a history of surgery or radiotherapy on the ipsilateral breast, a Karnofsky score $<70$, pregnancy or lactation, and contra-indications to either MRI or administration of a gadolinium-based contrast agent.

Examinations at $7 \mathrm{~T}$ and $3 \mathrm{~T}$ were performed on separate days due to the administration of the contrast agent. In pre-menopausal patients, MRI was performed between days 6 and 13 of the menstrual cycle.

Both prospective studies were approved by the Institutional Review Board of the University Medical Center Utrecht, the Netherlands. Written informed consent was obtained from all patients before participation.

\section{Data acquisition}

The 7T scans were performed on a whole-body scanner (Philips Healthcare, Cleveland, OH, USA). A unilateral two-channel (Stehouwer et al. 2013a, b; van de Bank et al. 2013) transmit/receive breast coil (MR Coils, Drunen, the Netherlands) was used for the first group. A bilateral four-channel (Italiaander and NPKOea 2012) coil (MR Coils, Drunen, the Netherlands) was used for the second group. The scan protocol included DCE (dynamic contrast-enhanced) imaging and an ultra-high resolution sequence. The DCE series was constructed using conventional imaging parameters comparable to $3 \mathrm{~T}$ MRI. They consisted of seven consecutive 3D T1w gradient echo (GRE) sequences with fat suppression [TR/ TE $5 / 2 \mathrm{~ms}$, binomial nominal flip angle (FA) $15^{\circ}$, FOV $160 \times 160 \times 160 / 350$ (unilateral/bilateral) $\mathrm{mm}^{3}$, acquired resolution $1 \times 1 \times 2 \mathrm{~mm}^{3}$, temporal resolution $63 / 67 \mathrm{~s}$.] with the administration of $0.1 \mathrm{mmol} / \mathrm{kg}$ gadobutrol (Gd, Bayer Schering Pharma AG, Berlin, Germany). Ultrahigh resolution imaging was performed using a T1w 3D GRE sequence with spectrally selective adiabatic inversion recovery (SPAIR) fat suppression [TR/TE/TI $7.0 / 2.9 / 120 \mathrm{~ms}$, FA $12^{\circ}$, FOV $120 \times 120 \times 120 / 350 \mathrm{~mm}^{3}$, acquired resolution $0.5 \mathrm{~mm}$ isotropic].

For the first patient group, the 3T scans were performed on a whole-body scanner (Philips Healthcare, Best, the Netherlands), using a dedicated seven-channel receiveonly breast coil (MRI devices, Würzburg, Germany). We used the hospital's clinical tumor detection and staging protocol. This protocol included a T2-weighted (T2w) sequence, DCE imaging, and a high resolution sequence. The dynamic series consisted of six consecutive 3D T1w GRE sequences with SPAIR fat suppression [TR/TE/ TI $3.1 / 1.17 / 90 \mathrm{~ms}$, FA $10^{\circ}$, FOV $360 \times 360 \times 150 \mathrm{~mm}^{3}$, 

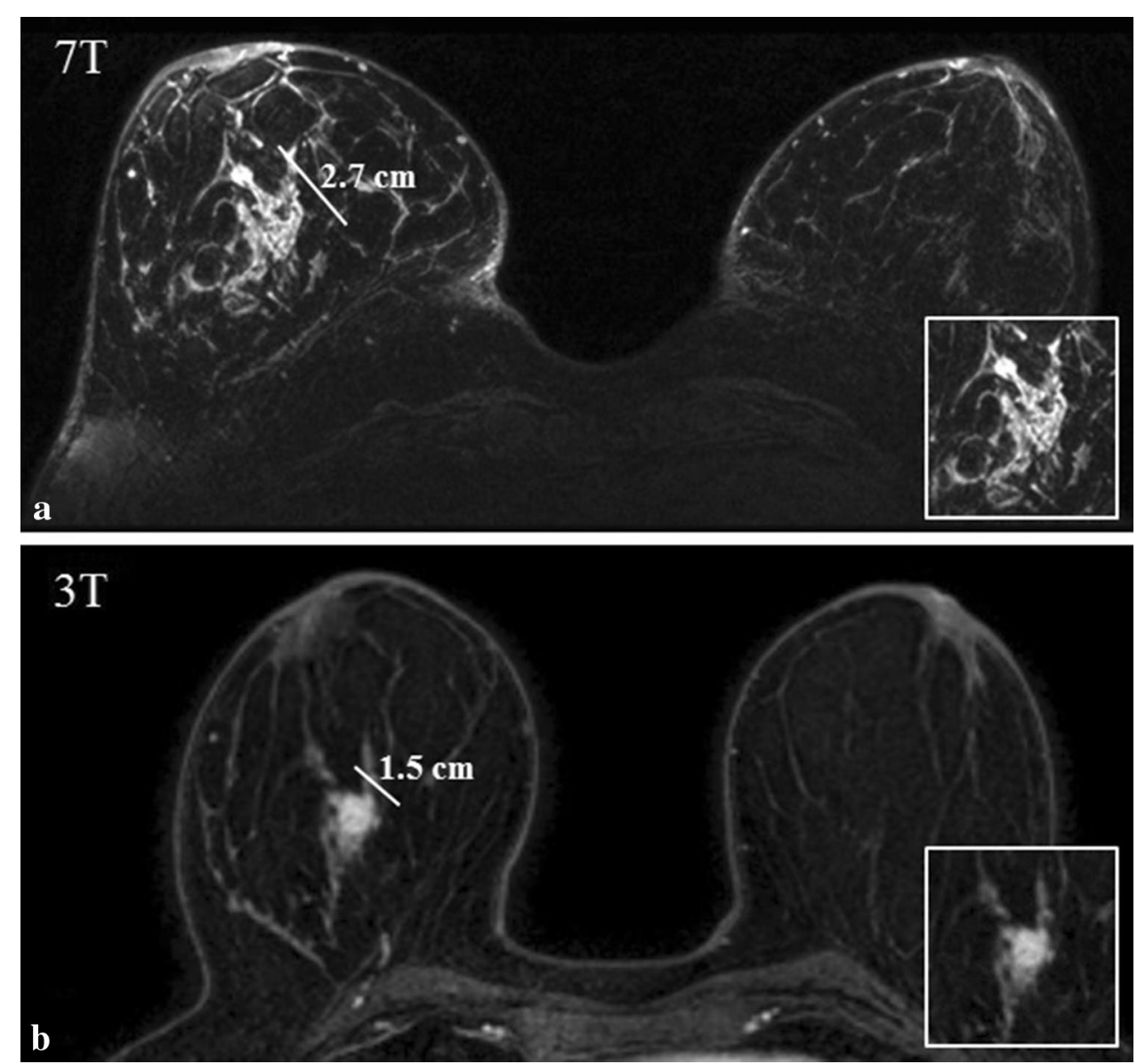

Fig. $47 T$ (a) and $3 T$ (b) images of a 52-year-old female patient diagnosed with a ductal carcinoma in her right breast. The morphological characteristics, the size and the borders of the lesion are more clearly depicted in ultra-high resolution 7T image (a) when compared to 3T images (b). This variation in tumor size has an impact in both staging and treatment of breast tumors. Both observers agreed on the sizes of the lesion

acquired resolution $1.1 \times 1.1 \times 2.4 \mathrm{~mm}^{3}$, temporal resolution $60 \mathrm{~s}$ ], and with the administration of $0.1 \mathrm{mmol} /$ $\mathrm{kg}$ gadobutrol. High resolution imaging was performed directly following the dynamic series [TR/TE/TI $4.5 / 1.67 / 90 \mathrm{~ms}$, FA $10^{\circ}$, FOV $360 \times 380 \times 180$, acquired resolution $0.65 \times 0.65 \times 2.00 \mathrm{~mm}^{3}$ ]. For the second patient group the protocol remained the same, except for the 3T dynamic series that was updated to obtain an acquired resolution of $0.9 \times 0.92 \times 1.80 \mathrm{~mm}^{3}$. Therefore, the previous high resolution sequence was discarded.

\section{Image analysis}

Two observers (R1 and R2) independently rated all examinations. $\mathrm{R} 1$ was a breast radiologist with 8 years of experience in breast MRI, and R2 a radiologist with 3 years of experience. Both observers were blinded for the histopathological results.

We performed imaging analysis with Aegis breast software (Hologic Inc. MA), which enabled the observers to assess kinetic curve information and to use a colorcoded overlay showing the different levels of initial and late enhancement. For the first group (with unilateral 7T images), the breast of interest was pointed out to the observers when assessing the 3T examination (for fair comparison).

First, image quality was rated for the dynamic series on a 5-point scale based on the following:

1. Excellent: no or hardly perceivable signal intensity variations across the field of view, i.e. homogeneous $\mathrm{B} 1+$ field, no or only mild artifacts, homogeneous fat suppression, and high visual SNR.

2. Good: mild heterogeneity changes in signal intensity across the field of view, e.g. mild gradual signal decrease from nipple towards chest wall, mild artifacts, mild inhomogeneity in fat suppression and high visual SNR. 
3. Moderate: moderate $\mathrm{B} 1+$ inhomogeneity and/or moderate presence of artifacts and/or moderate inhomogeneous fat suppression and a high visual SNR.

4. Poor: insufficient signal intensity homogeneity, with substantial signal intensity variations across the field of view and/or substantial image degradation due to artifacts, substantial inhomogeneity of fat suppression and/or a low visual SNR.

5. Non-diagnostic: insufficient quality for diagnosis because of insufficient signal homogeneity or a complete loss of signal intensity in parts of the FOV owing to severe artifacts, severe inhomogeneity of fat suppression and/or poor visual SNR.

Second, all identified lesions were classified according to the first edition of the BI-RADS lexicon (2003) as proposed by the American College of Radiology (Molleran and Mahoney 2010). The maximal diameter of the identified mass lesions was measured.

Lastly, contrast-enhancement-to-noise ratios (CENRs) were calculated for mass lesions. Calculations were conducted by comparing the ratio of the signal intensity of the lesions and the standard deviation of the signal in a homogenous area of tissue adjacent to the lesion. This comparison was made between the pre and post-contrast administration images (Stehouwer et al. 2013a), resulting in a measurement that showed the lesion conspicuity.

\section{Statistics}

Analyses were performed on a per examination basis. Image quality scores of both field strengths were compared using the Wilcoxon matched pairs signed rank test. Sensitivity, specificity, negative predictive value (NPV), and positive predictive value (PPV) with $95 \%$ confidence intervals (CI) were calculated using dichotomized classification scores (lesions classified as BI-RADS 1-3 were considered benign, and the ones classified as 4-5 were considered malignant). The same scores were used to calculate inter-reader agreement of BI-RADS classification assessed with $\mathrm{K}$ statistics. Histopathology was the reference standard. Sizes of malignant masses and CENRs were compared between field strengths using Wilcoxon matched pairs signed rank test. All analyses were performed using SPSS 20.0 (IBM Corp., NY, USA).

\footnotetext{
Authors' contributions

GLGM, BLS, DWJK, TAV, MAAJB, FMK, VOB, WJMK, PRL, WBV conceived the study, helped with data interpretation, participated in the study design and helped to draft the manuscript. GLGM, BLS and FMK contributed with data acquisition. GLGM and BLS coordinated the study, drafted the manuscript and contributed with data analysis and interpretation. All authors read and approved the final manuscript.
}

\section{Acknowledgements}

Financial support has been provided by A Sister's Hope/Pink Ribbon.

\section{Competing interests}

The authors declare that they have no competing interests.

Received: 16 December 2015 Accepted: 21 December 2015

Published online: 05 January 2016

\section{References}

Boer VO, van de Bank BL, Van VG et al (2012) Direct BO field monitoring and real-time $B 0$ field updating in the human breast at 7 Tesla. Magn Reson Med. 67(2):586-591

Brown R, Storey P, Geppert C et al (2013) Breast MRI at 7 Tesla with a bilateral coil and T1-weighted acquisition with robust fat suppression: image evaluation and comparison with 3 Tesla. Eur Radiol. 23(11):2969-2978

Brown R, Storey P, Geppert C et al (2014) Breast MRI at 7 Tesla with a bilateral coil and robust fat suppression. J Magn Reson Imaging 39(3):540-549

Edwards SD, Lipson JA, Ikeda DM et al (2013) Updates and revisions to the BI-RADS magnetic resonance imaging lexicon. Magn Reson Imaging Clin N Am 21(3):483-493

Gruber S, Pinker K, Zaric O et al (2014) Dynamic contrast-enhanced magnetic resonance imaging of breast tumors at 3 and $7 \mathrm{~T}$ : a comparison. Invest Radiol 49(5):354-362

Italiaander MG, NPKOea (2012) High B1 dutycycle in bilateral breast imaging at 7T. In: Proceedings of the 20th Annual Meeting of ISMRM, Melbourne, Australia, pp 1493 (Ref Type: Generic)

Klomp DW, van de Bank BL, Raaijmakers A et al (2011) 31P MRSI and $1 \mathrm{H} \mathrm{MRS} \mathrm{at}$ 7T: initial results in human breast cancer. NMR Biomed 24(10):1337-1342

Korteweg MA, Veldhuis WB, Visser F et al (2011) Feasibility of 7 Tesla breast magnetic resonance imaging determination of intrinsic sensitivity and high-resolution magnetic resonance imaging, diffusion-weighted imaging, and (1)H-magnetic resonance spectroscopy of breast cancer patients receiving neoadjuvant therapy. Invest Radiol 46(6):370-376

Kuhl CK, Schild HH, Morakkabati N (2005) Dynamic bilateral contrastenhanced MR imaging of the breast: trade-off between spatial and temporal resolution. Radiology. 236(3):789-800

Le-Petross H, Kundra V, Szklaruk J et al (2010) Fast three-dimensional dual echo dixon technique improves fat suppression in breast MRI. J Magn Reson Imaging 31(4):889-894

Loo CE, Straver ME, Rodenhuis S et al (2011) Magnetic resonance imaging response monitoring of breast cancer during neoadjuvant chemotherapy: relevance of breast cancer subtype. J Clin Oncol 29(6):660-666

Ma J (2008) Dixon techniques for water and fat imaging. J Magn Reson Imaging 28(3):543-558

Menezes GL, Knuttel FM, Stehouwer BL et al (2014) Magnetic resonance imaging in breast cancer: a literature review and future perspectives. World J Clin Oncol 5(2):61-70

Molleran V, Mahoney MC (2010) The BI-RADS breast magnetic resonance imaging lexicon. Magn Reson Imag Clin N Am 18(2):171-185

Noebauer-Huhmann IM, Szomolanyi P, Juras V et al (2010) Gadolinium-based magnetic resonance contrast agents at 7 Tesla: in vitro T1 relaxivities in human blood plasma. Invest Radiol 45(9):554-558

Peters NH, Borel Rinkes IH, Zuithoff NP et al (2008) Meta-analysis of MR imaging in the diagnosis of breast lesions. Radiology 246(1):116-124

Pinker K, Grabner G, Bogner W et al (2009) A combined high temporal and high spatial resolution 3 Tesla MR imaging protocol for the assessment of breast lesions: initial results. Invest Radiol 44(9):553-558

Pinker K, Bogner W, Baltzer P et al (2014) Clinical application of bilateral high temporal and spatial resolution dynamic contrast-enhanced magnetic resonance imaging of the breast at 7 T. Eur Radiol. 24(4):913-920

Rahbar H, Partridge SC, DeMartini WB et al (2013) Clinical and technical considerations for high quality breast MRI at 3 Tesla. J Magn Reson Imaging 37(4):778-790

Rahbar H, DeMartini WB, Lee AY et al (2015) Accuracy of 3 T versus 1.5 T breast MRI for pre-operative assessment of extent of disease in newly diagnosed DCIS. Eur J Radiol 84(4):611-616 
Soher BJ, Dale BM, Merkle EM (2007) A review of MR physics: 3T versus 1.5T. Magn Reson Imaging Clin N Am 15(3):277-290

Stehouwer BL, Klomp DW, Korteweg MA et al (2013a) 7 T versus 3T contrastenhanced breast magnetic resonance imaging of invasive ductulolobular carcinoma: first clinical experience. Magn Reson Imaging. 31(4):613-617 Stehouwer BL, Klomp DW, van den Bosch MA et al (2013b) Dynamic contrastenhanced and ultra-high-resolution breast MRI at 7.0 Tesla. Eur Radiol 23(11):2961-2968

Stehouwer BL, van der Kemp WJ, Luijten PR et al (2014) (31) P magnetic resonance spectroscopy of the breast and the influence of the menstrual cycle. Breast Cancer Res Treat. 144(3):583-589

Umutlu L, Kraff O, Orzada S et al (2011) Dynamic contrast-enhanced renal MRI at 7 Tesla: preliminary results. Invest Radiol 46(7):425-433 van de Bank BL, Voogt IJ, Italiaander M et al (2013) Ultra high spatial and temporal resolution breast imaging at 7T. NMR Biomed 26(4):367-375

van der Kemp WJ, Stehouwer BL, Luijten PR et al (2014) Detection of alterations in membrane metabolism during neoadjuvant chemotherapy in patients with breast cancer using phosphorus magnetic resonance spectroscopy at 7 Tesla. Springerplus 3(634)

van der Velden TA, Italiaander M, van der Kemp WJ et al (2014), Radiofrequency configuration to facilitate bilateral breast P MR spectroscopic imaging and high-resolution MRI at 7 Tesla. Magn Reson Med

van Kalleveen IM, Boer VO, Luijten PR et al (2015) Tilt optimized flip uniformity (TOFU) RF pulse for uniform image contrast at low specific absorption rate levels in combination with a surface breast coil at 7 Tesla. Magn Reson Med. 74(2):482-488

\section{Submit your manuscript to a SpringerOpen ${ }^{\odot}$ journal and benefit from:}

- Convenient online submission

- Rigorous peer review

- Immediate publication on acceptance

- Open access: articles freely available online

- High visibility within the field

- Retaining the copyright to your article

Submit your next manuscript at $>$ springeropen.com 